\title{
Feasibility of Personal Sound Amplification Products in Patients With Moderate Hearing Loss: A Pilot Study
}

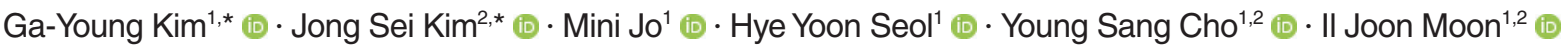 \\ ${ }^{1}$ Hearing Research Laboratory, Samsung Medical Center, Seoul; ${ }^{2}$ Department of Otorhinolaryngology-Head and Neck Surgery, \\ Samsung Medical Center, Sungkyunkwan University School of Medicine, Seoul, Korea
}

Objectives. This study was conducted to investigate the electroacoustic characteristics of personal sound amplification products (PSAPs), to identify whether PSAPs provide adequate gain and output for three common hearing loss (HL) configurations, and to compare the benefits of a representative PSAP (RPSAP) and a conventional hearing aid (HA) for clinical hearing outcomes as a pilot study.

Methods. The study comprised three phases: electroacoustic analysis, simulated real-ear measurements (REMs), and clinical hearing experiments. Electroacoustic analysis and simulated REMs were performed for three basic PSAPs (BeethoSOL, EarJJang, and Geniesori2) and three high-end PSAPs (Hearing Able, Olive Smart Ear, and Soriln) using the Aurical Hearing Instrument Test box with a 2-mL coupler. Four electroacoustic characteristics (maximum output sound pressure level at $90 \mathrm{~dB}$ SPL, frequency range, equivalent input noise, and total harmonic distortion) were investigated. By simulated REMs, appropriate levels of the six PSAPs for three common HL configurations (mild-to-moderate high-frequency HL, moderate to moderately severe sloping HL, and moderate flat HL) were determined. Clinical experiments compared the performance of RPSAP to HA, both of which were fitted by audiologists using REMs. Clinical experiments were administered using functional gain, a word recognition test, and the Korean version of the Hearing in Noise Test in six participants with bilateral moderate sensorineural HL.

Results. The two high-end devices met all tolerances. One basic and two high-end PSAPs showed appropriate levels for three common HL configurations. In the clinical experiments, the RPSAP showed better performance than unaided, but slightly worse than HA under all test conditions.

Conclusion. Certain PSAPs met all specified tolerances for electroacoustic analysis and approximated prescriptive targets in well-controlled laboratory conditions. The pilot clinical experiments explored the possibility that the RPSAP could serve as a hearing assistive device for patients with moderate HL.

Keywords. Hearing Loss; Hearing Aids; Sensorineural Hearing Loss; Wearable Electronic Devices

\section{INTRODUCTION}

- Received November 25, 2020

Revised January 26, 2021

Accepted January 27, 2021

- Corresponding author: Il Joon Moon

Department of Otorhinolaryngology-Head and Neck Surgery, Samsung

Medical Center, Sungkyunkwan University School of Medicine,

81 Irwon-ro, Gangnam-gu, Seoul 06351, Korea

Tel: +82-2-3410-3879, Fax: +82-2-3410-3579

E-mail:moon.iljoon@gmail.com

*These authors contributed equally to this work as first authors.
According to World Health Organization estimates, over 5\% of the world's population is affected by hearing loss, and this proportion is expected to increase to one in every 10 people by 2050 [1]. Untreated hearing loss is a major health and social problem. Moreover, because hearing loss can be associated with diminished cognitive function [2], proactive hearing rehabilitation is needed for people with hearing loss.

The uptake rate of hearing aids (HAs) in patients with hearing

Copyright $@ 2022$ by Korean Society of Otorhinolaryngology-Head and Neck Surgery

This is an open-access article distributed under the terms of the Creative Commons Attribution Non-Commercial License (https://creativecommons.org/licenses/by-nc/4.0)

which permits unrestricted non-commercial use, distribution, and reproduction in any medium, provided the original work is properly cited. 
loss remains relatively low throughout the world despite the negative consequences of hearing loss. For instance, the uptake rate of HAs in Korea was $17.4 \%$ based on data from a national survey [3]. A study conducted by the Korean National Evidencebased Healthcare Collaborating Agency reported the following reasons: inconvenience of wearing HAs (49.1\%), costs of HA purchase and maintenance (46.4\%), and stigma associated with HAs (37.1\%) [4]. Cost is a critical barrier for HA acquisition. While the average cost for a HA is currently around $\$ 2,000$, individuals prefer to pay less than the average cost when purchasing the devices [4].Thus, over-the-counter (OTC) hearing devices, including personal sound amplification products (PSAPs), may have the potential to serve as less expensive alternatives to HAs.

To accommodate this rise in interest, in the United States, the President's Council of Advisors on Science and Technology recommended that the Food and Drug Administration (FDA) should approve hearing devices for OTC sale to promote access to hearing technology for people with hearing loss [5].The FDA planned to develop regulations for publicly available OTC products for consumers with mild to moderate hearing loss by 2020 [6]. However, under current regulations, PSAPs should not be advertised as devices that can compensate for hearing loss.

PSAPs, as OTC hearing devices, were originally designed to amplify ambient sounds for hunting, bird watching, and listening to lectures at a long distance. However, these devices have recently been used to manage hearing loss. Several studies have shown that PSAPs may serve as alternatives to conventional HAs for mild to moderate hearing loss [7-9]. However, those previous studies investigated PSAPs that were not prescribed using best-practice protocols, such as real-ear measurements (REMs). All hearing assistive devices perform best when appropriately fitted by hearing experts. Therefore, it is important to properly fit PSAPs used for hearing compensation. The objectives of this study were three-fold: (1) to investigate the electroacoustic characteristics of PSAPs, (2) to identify whether PSAPs provide adequate gain and output for three common hearing loss configurations, and (3) to compare the benefit of a representative PSAP (RPSAP) to that of a conventional HA for clinical hearing outcomes as a pilot study.

\section{HII G H L I G H T S}

- High-end personal sound amplification products (PSAPs) satisfied most of the tolerances for specifying electroacoustic data.

- PSAPs showed sufficient amplification towards the prescriptive targets when their gain could be adjusted across frequencies through a smartphone application.

- The pilot clinical hearing experiments explored the possibility that PSAPs could serve as hearing assistive devices for patients with moderate hearing loss.

\section{MATERIALS AND METHODS}

This study was approved by the Institutional Review Board of Samsung Medical Center in Seoul (IRB No. SMC 2020-05-052001), South Korea in accordance with the Declaration of Helsinki. All patients signed a written informed consent form prior to enrollment.

\section{Phase 1. electroacoustic analysis}

Six PSAPs were selected based on the following inclusion criteria:The PSAPs needed to (1) be distributed in Korea, (2) be readily accessible to the public, and (3) have measurable electroacoustic characteristics. The PSAPs were divided into two price groups based on the retail cost for a pair: a basic group consisting of three devices (BeethoSOL, EarJJang, and Geniesori2) and a high-end group consisting of three devices (Hearing Able, Olive Smart Ear, and SoriIn). All PSAPs used in this study are presented in Table 1.

Electroacoustic measurements were performed for all devices using the Aurical Hearing Instrument Test (HIT) box and the OTOsuite software (GN Otometrics A/S, Taastrup, Denmark). An HA-1 2-mL coupler was used to measure all devices, except for one device. One BTE (behind the ear) device (Soriln) was analyzed using an HA-2 coupler.

Tolerances for specifying electroacoustic data were established based on previous studies $[10,11]$. Maximum output sound pressure level at $90 \mathrm{~dB}$ SPL (OSPL $90 \mathrm{Max}$ ) represents the maximum output of a device when the input SPL is $90 \mathrm{~dB}$ with the gain control of the device set to its full-on position. The OSPL 90 Max tolerance was set to be no greater than $120 \mathrm{~dB}$ SPL. The frequency range refers to the range between the minimum and maximum frequencies given an input of $60 \mathrm{~dB}$ SPL with the reference test setting of the gain control (RTS). The frequency range includes the speech frequency range of $250-6,000 \mathrm{~Hz}$. The equivalent input noise (EIN) refers to the noise produced by the device during amplification with the gain control in the RTS. A tolerance of EIN was less than $28 \mathrm{~dB}$ SPL. The total harmonic distortion (THD) is measured at the gain control in the RTS using a puretone input signal at three different frequencies: $70 \mathrm{~dB}$ SPL at $500 \mathrm{~Hz}$ and $800 \mathrm{~Hz}$ and $65 \mathrm{~dB}$ SPL at 1,600 Hz. The THD was not to exceed $3 \%$ at any given frequency.

\section{Phase 2. simulated REMs}

In the second phase of the study, simulated REMs were performed with a 2-mL coupler. Three common configurations of hearing loss (mild-to-moderate high-frequency hearing loss, moderate to moderately severe sloping hearing loss, and moderate flat hearing loss) were used according to previous study [10].The devices were placed in the Aurical HIT box and aided responses were measured at moderate input signal of $65 \mathrm{~dB}$ SPL. The appropriate level of the devices was adjusted to match as closely as possible the National Acoustics Laboratories-Non-Linear prescription, 2nd generation (NAL-NL2) targets. The appropriate level 


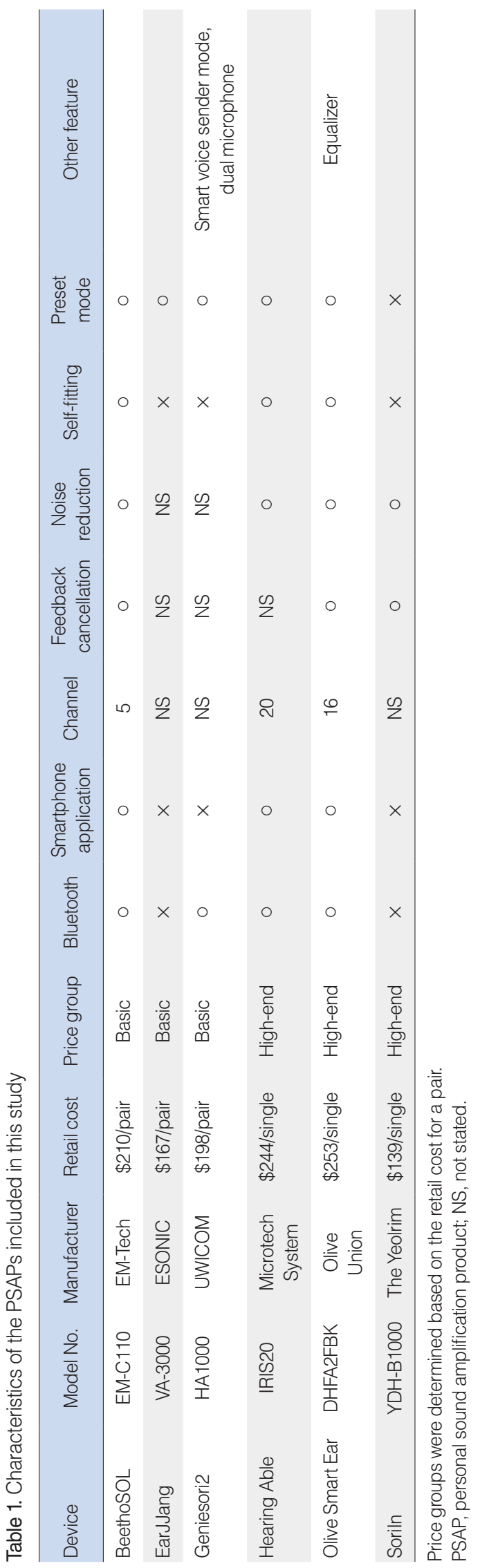

of device was determined by whether the difference between the NAL-NL2 target and aided response at seven frequencies $(0.25,0.5,1,2,3,4$, and $6 \mathrm{kHz})$ was within $10 \mathrm{~dB}$ SPL. Five out of seven frequencies or above was considered "appropriate level" for that hearing threshold [10].

\section{Phase 3. clinical outcomes}

Lastly, we conducted clinical experiments to identify the utility of one RPSAP by comparing their performance to a conventional HA for patients with bilateral moderate hearing loss (pure tone average of the four-frequency averages at $0.5,1.0,2.0$, and $4.0 \mathrm{kHz}$, 41-55 dB of hearing loss). The HA used were ReSound LiNX 3D LT962-DRW, denoted as the HA. Olive Smart Ear, denoted as the RPSAP, was selected as the RPSAP for these experiments based on three reasons. First, the RPSAP met all tolerances in electroacoustic analysis. Second, the RPSAP showed appropriate gains for three common hearing loss configurations. Third, the RPSAP could adjust gains across frequencies using a corresponding smartphone application. Before conducting clinical experiments, both the HA and RPSAP were fitted by audiologists using REMs. The RPSAP had a corresponding smartphone application that allows users to adjust the device's frequency response and volume. The test was first performed in unaided condition, and then the HA and RPSAP were put on in order and measured immediately without acclimatization period.

Six participants (one man and five women) were recruited from the outpatient clinic of the department of otolaryngology. Individuals who met the following eligibility criteria were included: patients who are under 70 years of age, who have bilateral moderate sensorineural hearing loss, and who are judged to have no abnormalities in the eardrum through otoscopy with a type A tympanogram. The mean age of the participants was 58.17 years (standard deviation, 6.18). The individual audiogram of each six patients were described in Fig. 1. All but one participant (subject 5) had no experience wearing HAs. The demographics and characteristics of the individual patients were shown in Table 2 .

Clinical experiments were administered using functional gain, a word recognition test, and the Korean version of the Hearing in Noise Test (K-HINT). Functional gain is defined as the difference in $\mathrm{dB}$ HL between aided and unaided sound-field thresholds at test frequencies $(0.25,0.5,1,2,3,4$, and $6 \mathrm{kHz})$. The results of functional gain testing are equal to real-ear insertion gain [12]. Functional gain was assessed using a front loudspeaker in a quiet sound-treated booth. Aided sound-field thresholds using FM (frequency modulation) signals centered at 250, 500, 1,000, 2,000, 3,000, 4,000, 6,000, and $8,000 \mathrm{~Hz}$ were obtained using the ASHA-recommended procedure [13]. Subjects were seated in a sound booth $1 \mathrm{~m}$ from a loudspeaker at $0^{\circ}$ azimuth. All tests were performed at bilateral aided state. The functional gain is obtained by subtracting the unaided threshold from the aided threshold. Unaided thresholds are measured to tones presented through the loudspeaker $1 \mathrm{~m}$ front of the patient. Aided thresh- 
olds are made using the same stimuli. The differences between the two represent the functional gain provided by HA and PSAP.

The word recognition test in sound-field was measured at 50 $\mathrm{dB} H \mathrm{HL}$ to obtain word recognition scores (percentage of correct recognition). Participants were asked to repeat 25 words from one of the Korean Standard-Monosyllabic Word Lists for Adults [14]. K-HINT [15] was used to measure reception thresholds for speech both in quiet and in front noise conditions. The unit of
A

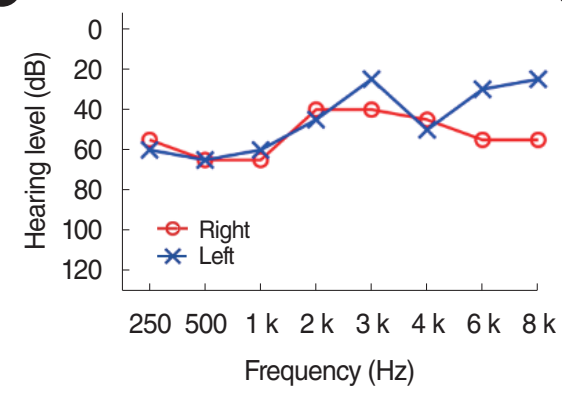

(D)

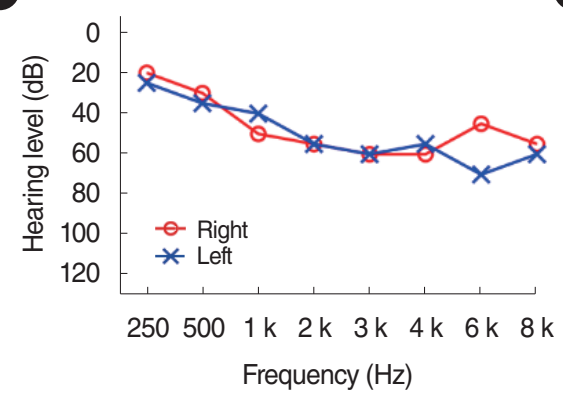

B

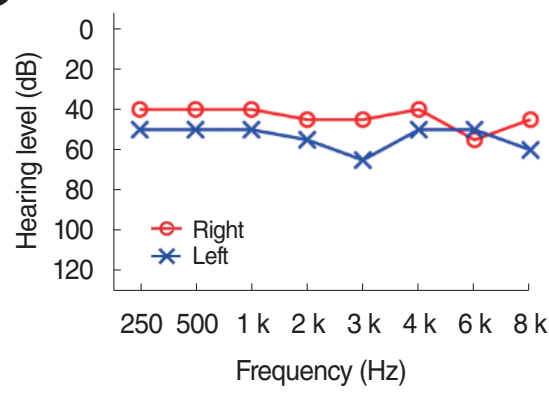

$\boldsymbol{E}$

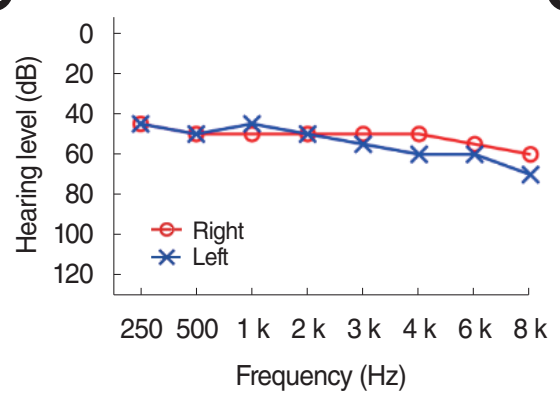

C

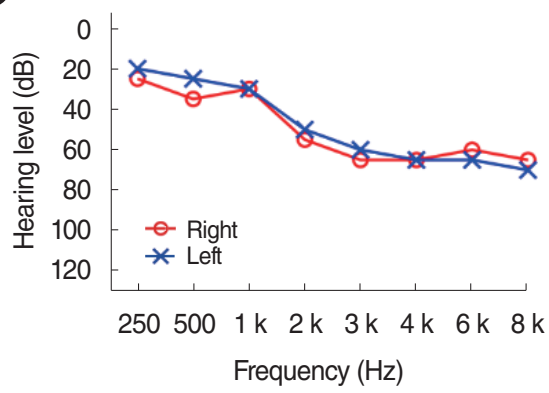

$\boldsymbol{F}$

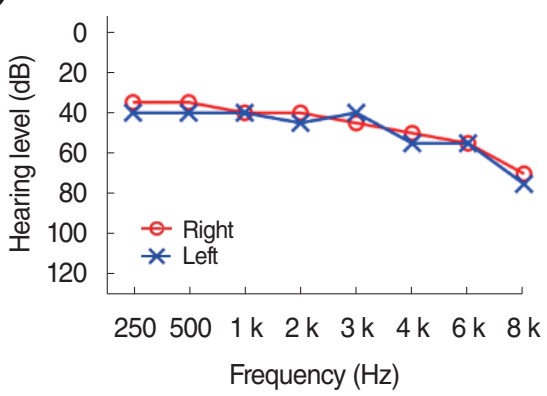

Fig. 1. (A-F) Individuals audiograms of the participants.

Table 2. Demographic and audiological characteristics of the individual patients

\begin{tabular}{lcccccccc}
\hline Patient No. & Sex & Age $(\mathrm{yr})$ & PTA $^{\text {a) }}$ (right) & PTA $^{\text {a) }}$ (left) & WRS (right) & WRS (left) & Tinnitus & HA user \\
\hline 1 & F & 60 & 53.75 & 55.00 & 80 & 76 & Both & No \\
2 & F & 56 & 41.25 & 51.25 & 100 & 98 & Both & No \\
3 & F & 51 & 46.25 & 42.50 & 88 & 96 & Both & No \\
4 & M & 68 & 48.75 & 46.25 & 92 & 88 & None & No \\
5 & F & 53 & 50.00 & 51.25 & 96 & 96 & Left & Yes \\
6 & F & 61 & 41.25 & 45.00 & 84 & 96 & Both & No \\
\hline
\end{tabular}

PTA, pure tone average; WRS, word recognition score; HA, hearing aid.

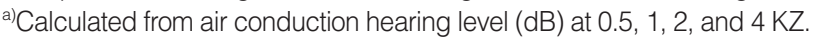

Table 3. Electroacoustic analysis of the PSAPs included in this study

\begin{tabular}{|c|c|c|c|c|c|c|}
\hline \multirow{3}{*}{ Device } & \multicolumn{6}{|c|}{ Electroacoustic analysis result } \\
\hline & \multirow{2}{*}{$\begin{array}{l}\text { OSPL90 Max } \\
\text { (dB SPL) }\end{array}$} & \multirow{2}{*}{$\begin{array}{c}\text { Frequency range } \\
(\mathrm{Hz})\end{array}$} & \multirow{2}{*}{$\begin{array}{c}\text { EIN } \\
(\mathrm{dB} S P L)\end{array}$} & \multicolumn{3}{|c|}{ THD (\%) } \\
\hline & & & & $500 \mathrm{~Hz}$ & $800 \mathrm{~Hz}$ & $1,600 \mathrm{~Hz}$ \\
\hline BeethoSOL (basic) & 115.9 & $293-8,030^{a)}$ & $32.4^{\text {a) }}$ & 2.4 & $4.5^{\text {a) }}$ & 2.5 \\
\hline EarJJang (basic) & 119.6 & $100-7,178$ & $39.0^{\mathrm{a})}$ & 0.4 & 0.2 & 2.5 \\
\hline Geniesori2 (basic) & $123.7^{\mathrm{a})}$ & $100-7,735$ & $35.4^{\mathrm{a})}$ & 0.2 & 0.1 & 0.1 \\
\hline Hearing-Able (high-end) & 103.0 & $100-3,717^{\mathrm{a})}$ & 26.7 & 0.9 & 1.0 & 0.1 \\
\hline Olive Smart Ear (high-end) & 97.2 & $215-6,650$ & 27.4 & 1.8 & 0.6 & 0.1 \\
\hline Soriln (high-end) & 109.6 & $100-7,024$ & 26.9 & 0.7 & 0.5 & 0.1 \\
\hline
\end{tabular}

PSAP, personal sound amplification product; OSPL90 Max, maximum output sound pressure level at $90 \mathrm{~dB}$ SPL; EIN, equivalent input noise; THD, total harmonic distortion.

a)Indicates measured values that deviated from the tolerance defined in the Methods section. 


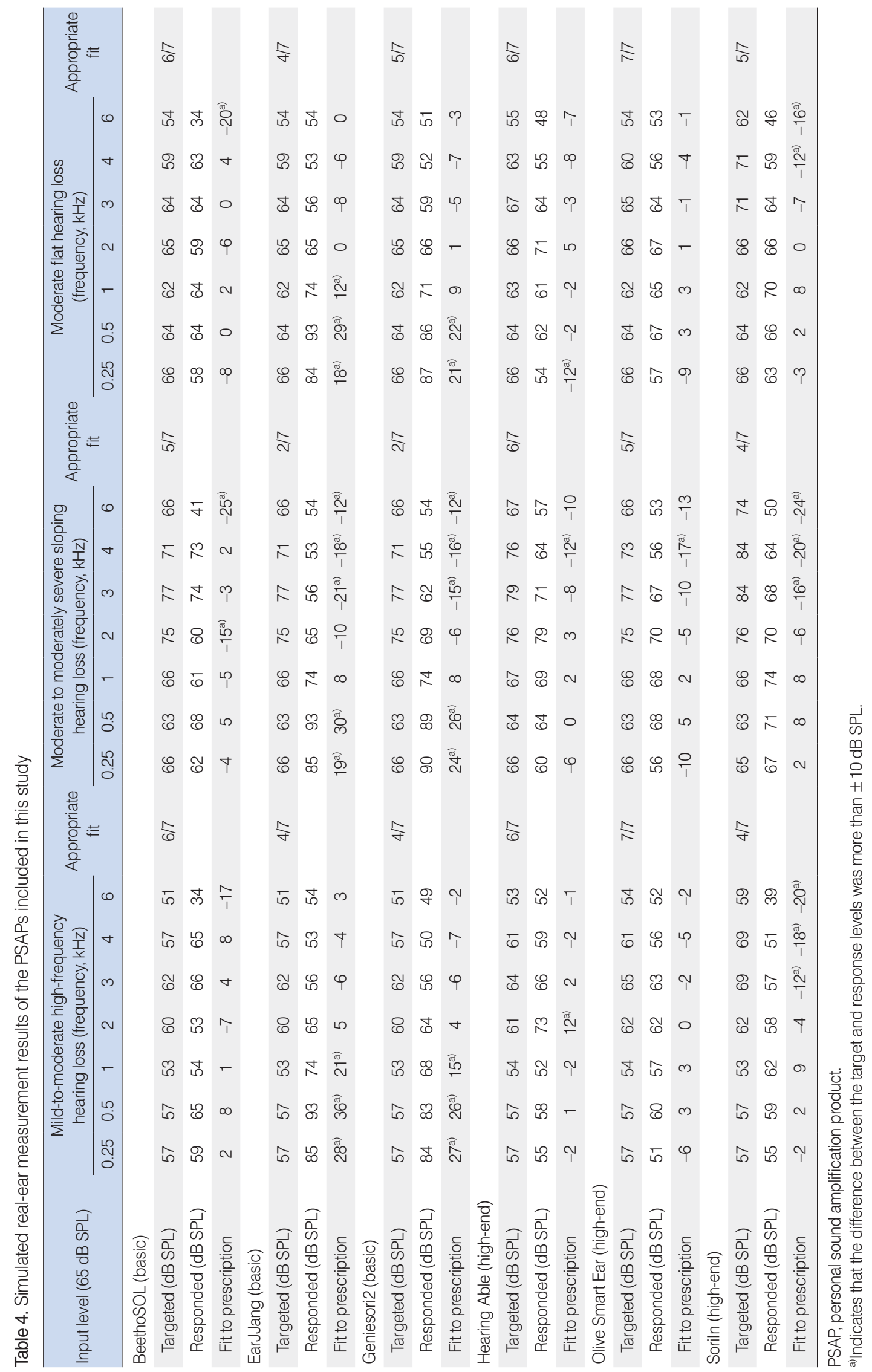


measure for threshold in the quiet condition is $\mathrm{dB}(\mathrm{A})$. The unit of measure for threshold in the noise condition is the $\mathrm{dB}$ signal-tonoise $(\mathrm{S} / \mathrm{N})$ ratio.

\section{Statistical analysis}

The statistical language $\mathrm{R}$ was used for the data analysis ver. 4.0.2 ( $\mathrm{R}$ foundation for Statistical Computing, Vienna, Austria). Wilcoxon signed-rank test was used to compare functional gain of the HA and PSAP.

\section{RESULTS}

\section{Phase 1. electroacoustic analysis}

Regarding OSPL 90 Max, five devices (BeethoSOL, EarJJang, Hearing Able, Olive Smart Ear, and SoriIn) were within the tolerance limits (<120 dB SPL). As for the frequency range, four (EarJJang, Geniesori2, Olive Smart Ear, and SoriIn) out of the six devices met the tolerance (250-6,000 Hz). For EIN, three devices (Hearing Able, Olive Smart Ear, SoriIn) were within the acceptance tolerance ( $<28 \mathrm{~dB}$ SPL). For THD, only the BeethoSOL device showed less satisfactory performance, generating a value of $3 \%$ or higher at $800 \mathrm{~Hz}(4.5 \%)$. In summary, two high- end devices (Olive Smart Ear and Soriln) met all tolerances. All results are presented in Table 3 .

\section{Phase 2. simulated REMs}

For mild-to-moderate high-frequency hearing loss, the output was not adequate for three devices. The two basic devices (EarJJang and Geniesori2) featured excessive amplification at low and middle frequencies. One high-end device (Soriln) displayed a lack of amplification at frequencies above $3 \mathrm{kHz}$. For the moderate to moderately severe sloping hearing loss, the basic devices exhibited a tendency to excessively amplify low frequencies and insufficient gain at high frequencies. The high-end devices also showed a lack of amplification at high frequencies. For moderate flat hearing loss, most devices showed adequate levels at all frequencies. However, one device (EarJJang) had excessive amplification in low and medium frequencies. In summary, one basic (BeethoSOL) and two high-end PSAPs (Hearing Able and Olive Smart Ear) showed appropriate levels for three common hearing loss configurations. All results are presented in Table 4.

\section{Phase 3. clinical outcomes}

Functional gain

The difference in functional gain between HA and the RPSAP

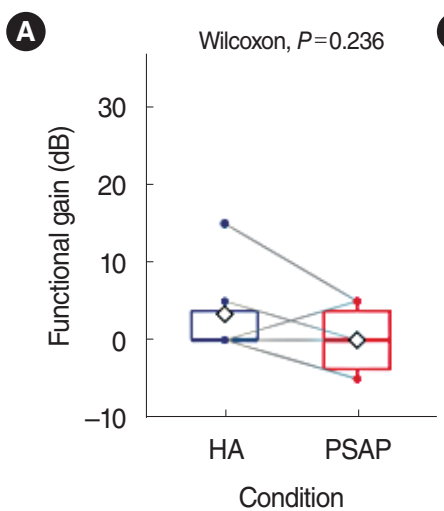

B
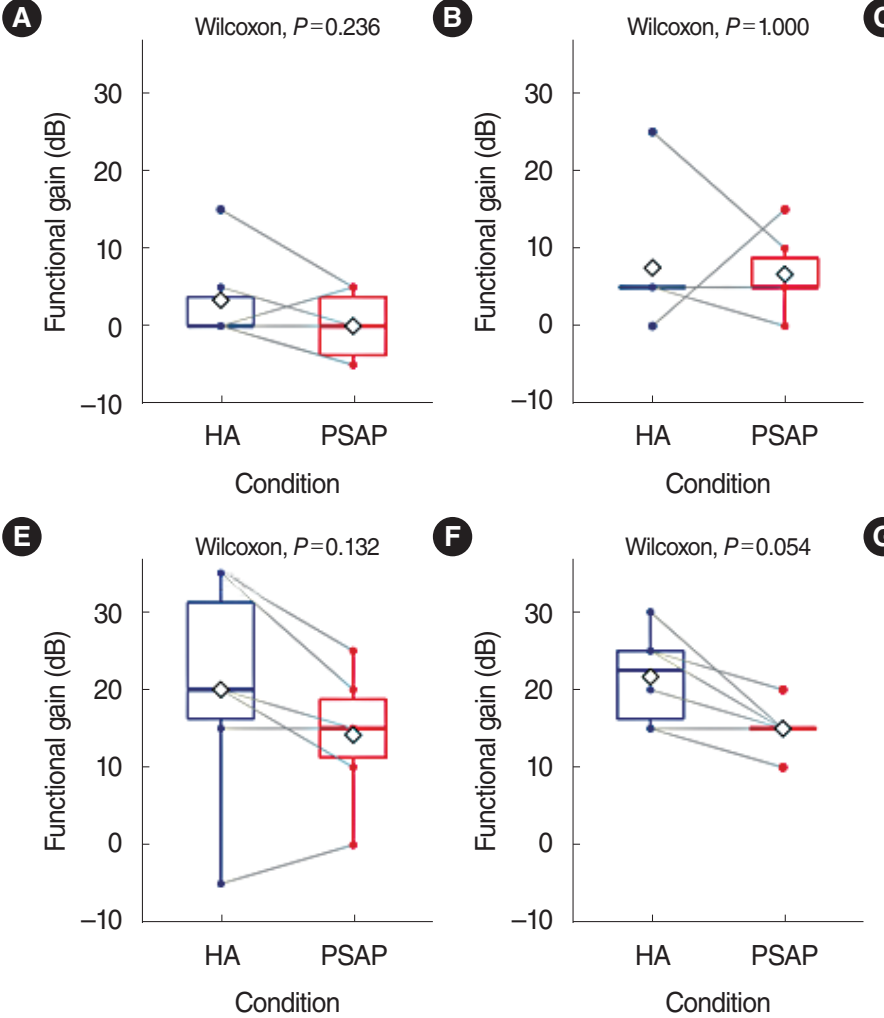
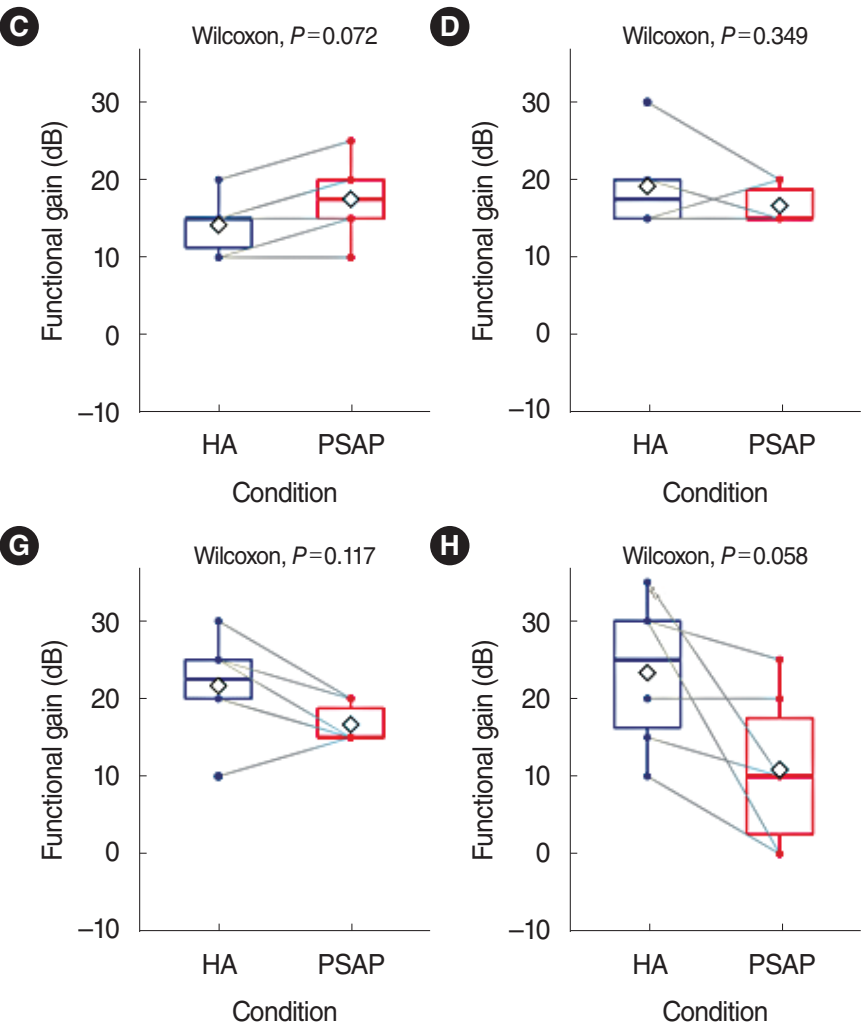

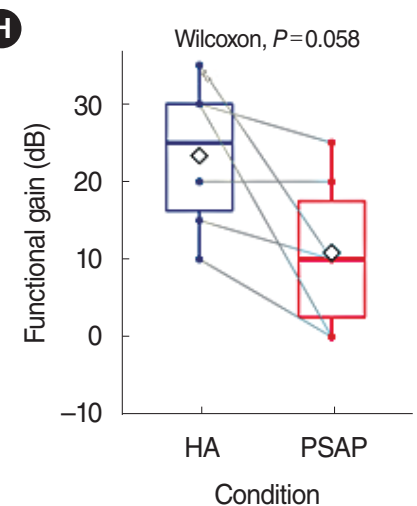

Fig. 2. Functional gain of a hearing aid $(H A)$ and a representative personal sound amplification product (RPSAP) for six participants by frequency: (A) $250 \mathrm{~Hz},(B) 500 \mathrm{~Hz},(\mathrm{C}) 1,000 \mathrm{~Hz},(\mathrm{D}) 2,000 \mathrm{~Hz},(\mathrm{E}) 3,000 \mathrm{~Hz},(\mathrm{~F}) 4,000 \mathrm{~Hz},(\mathrm{G}) 6,000 \mathrm{~Hz},(\mathrm{H}) 8,000 \mathrm{~Hz}$. Functional gain was defined as the difference in $\mathrm{dB} H \mathrm{H}$ between the aided and unaided thresholds. The overlapping dots and gray lines indicate individual changes. The rhombus shape indicates the mean values of the results. 
was analyzed, and no statistically significant differences were found between HA and the RPSAP in functional gain for any frequency. The functional gain was presented using a box plot with individual data in Fig. 2. Although not statistically significant, the HA showed better performance than the RPSAP un-
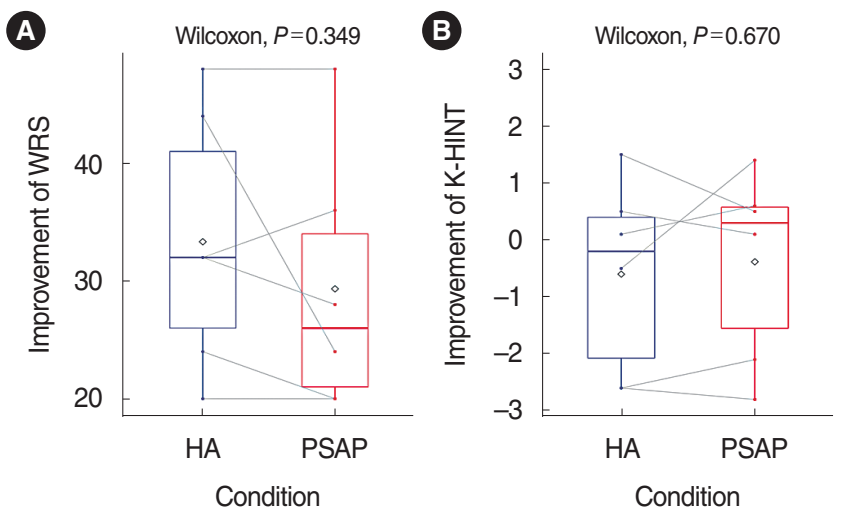

Fig. 3. Average results of the word recognition score (WRS; A) and Korean version of the Hearing in Noise Test (K-HINT; B) for the hearing aid $(\mathrm{HA})$ and representative personal sound amplification product (PSAP). The overlapping dots and gray lines indicate individual changes. The rhombus shape indicates the mean values of the results. der most conditions.

\section{Word recognition test}

The mean percentage score of the word recognition test was $48.7 \%$ in the unaided conditions, $82 \%$ in the HA conditions, and $78 \%$ in the RPSAP conditions. The difference between HA and RPSAP was not statistically significant (Fig. 3).

\section{The Korean version of the Hearing in Noise Test}

The mean threshold of K-HINT in quiet conditions was $46.4 \mathrm{~dB}(\mathrm{~A})$ in the unaided conditions, $36.9 \mathrm{~dB}(\mathrm{~A})$ in the HA conditions, and $38.9 \mathrm{~dB}(\mathrm{~A})$ in the RPSAP conditions. The mean threshold of $\mathrm{K}$ HINT in front noise conditions was $2.0 \mathrm{~dB} S / \mathrm{N}$ in the unaided conditions, $1.4 \mathrm{~dB}$ S/N in the HA conditions, and $1.6 \mathrm{~dB} S / \mathrm{N}$ in the RPSAP conditions. However, the difference between HA and RPSAP was not statistically significant (Fig. 3). Fig. 4 shows the K-HINT results for each participant.

\section{DISCUSSION}

This study was undertaken in order to explore the capabilities of PSAPs as communicative assistive devices and to provide evidence for appropriate recommendations to patients. The results
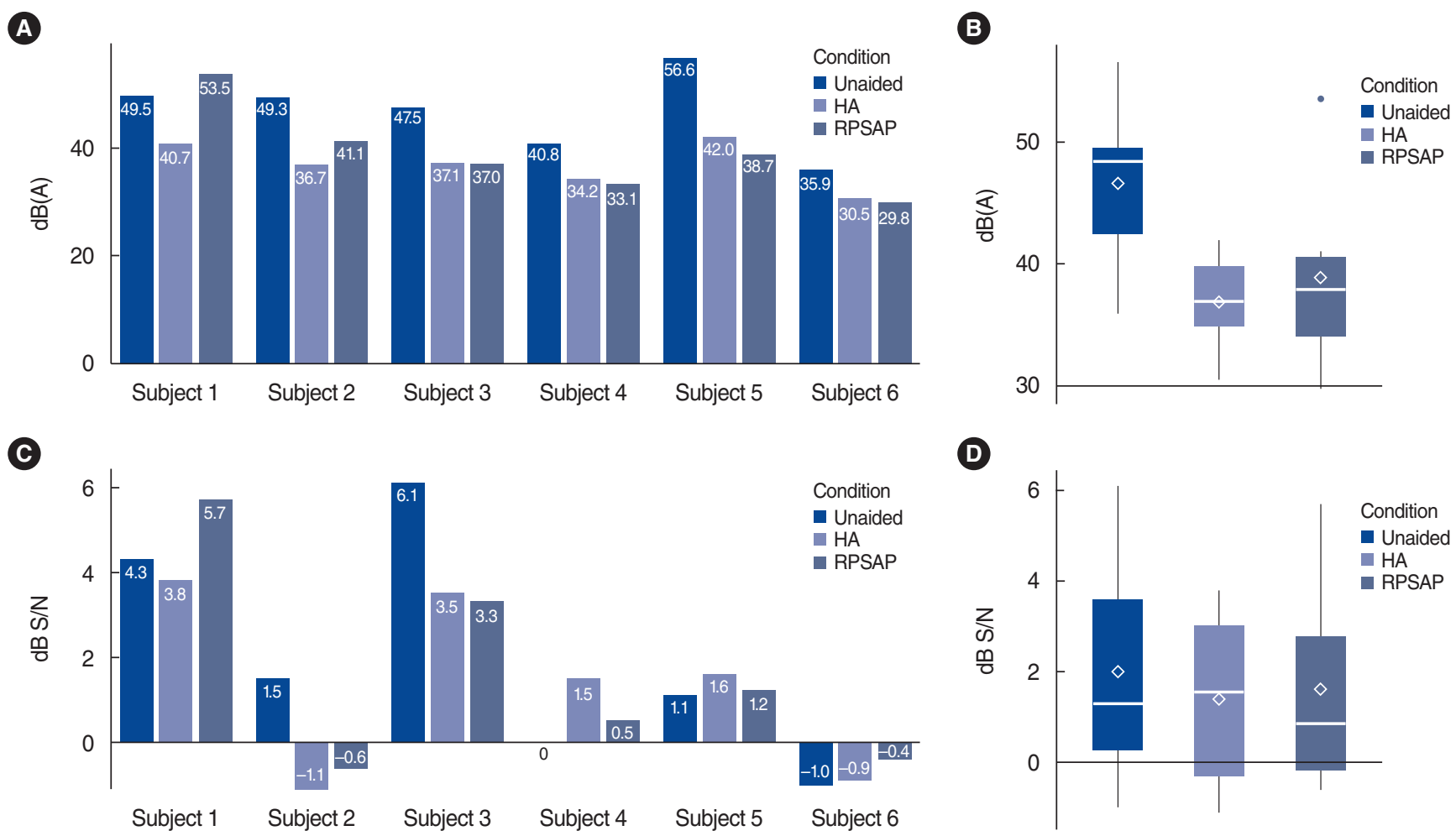

Fig. 4. Results of the Korean version of the Hearing in Noise Test (K-HINT) for each patient. (A) Individual results of the K-HINT in a quiet setting. (B) Average results for each condition of the K-HINT in a quiet setting. (C) Individual results of the K-HINT in a noisy setting. (D) Average results for each condition of the K-HINT in a noisy setting. The rhombus shape indicates the mean values of the results. HA, hearing aid; RPSAP, representative personal sound amplification product; S/N, signal-to-noise. 
demonstrated that certain PSAPs met all specified tolerances for electroacoustic measurements and had the ability to approximate target gain in well-controlled laboratory conditions. The pilot clinical experiments also explored the possibility that the RPSAP could serve as a hearing assistive device for patients with moderate hearing loss.

Our results indicated that high-end PSAPs are comparable to conventional HAs in terms of their electroacoustic testing results. All three high-end PSAPs performed within three or more of the defined tolerance limits. In contrast, the basic PSAPs did not meet most of the tolerances. The manufacturers presented their devices' electroacoustic information on their website. However, most of this information contained one or two components of electroacoustic testing and the terminology was inconsistent. Two devices (BeethoSOL and Olive Smart Ear) offered detailed information, but somewhat different results were obtained in the present study. For instance, the maximum output sound pressure level of the Olive Smart Ear was $112.8 \mathrm{~dB}$ SPL ( $\pm 3 \mathrm{~dB}$ SPL) in the manufacturer's specification sheet, but was $97.2 \mathrm{~dB}$ SPL in our study.

One possible reason for this discrepancy is that the manufacturer may have used different measurement methods. There is no international standard for the electroacoustic analysis of PSAPs $[10,11]$. In other words, the variation in the tested values might be due to heterogeneity in electroacoustic measurement procedures.

By simulating REMs, we confirmed that the gain of PSAPs was adjusted to the NAL-NL2 targets in line with three common hearing loss configurations. Regardless of the price and hearing loss configuration, the frequency responses were able to meet NAL-NL2 targets when the manufacturer provided a corresponding smartphone application. It is highly important to accurately adjust hearing devices in accordance with the user's auditory characteristics. Therefore, when considering a PSAP for auditory rehabilitation purposes, users need to confirm whether the PSAP has adjustable levels for each frequency. In moderate to moderately severe sloping hearing loss, the gain was insufficient for high frequencies, even when the difference between the aided response and the target was within the acceptable range $( \pm 10 \mathrm{~dB}$ SPL). These results are consistent with a prior study showing that the functionality of PSAPs was insufficient for moderately severe hearing loss [8]. Furthermore, two basic PSAPs provided excessive low-frequency gain, leading to problems with the discrimination of speech sounds due to the highly amplified noise [16].

Finally, we investigated whether the RPSAP was clinically suitable for patients with bilateral moderate hearing loss. For the word recognition test and K-HINT, the RPSAP and HA performed better than the unaided conditions, and the RPSAP performed similarly to the HA. These findings demonstrated that the RPSAP could improve communication in quiet and noisy conditions to a similar extent as HA for patients with moderate hear- ing loss. This was a preliminary study conducted to inform future research, and it is difficult to consider the clinical outcomes to be significant owing to the small sample size. However, many previous studies have reported that PSAPs could improve communication skills in patients with mild to moderate hearing loss [7-9]. In addition, the findings of our study are meaningful in that the clinical experiments were conducted after performing REMs for both the HA and RPSAP.

A number of studies have investigated various PSAPs in parallel with OTC hearing devices, and have considered PSAPs as a more affordable option for individuals with mild to moderate hearing loss [7-9]. To the best of our knowledge, this is the first study conducted in Korea to report that PSAPs can be considered as hearing assistive devices. Since most individuals with hearing loss are elderly [17], it is difficult for them to purchase PSAPs that are primarily sold overseas. Hence, a significant aspect of this study is that it demonstrated the electroacoustic properties and simulated REMs' outcomes of PSAPs readily available in Korea. Our results provide preliminary data for otolaryngologists, audiologists, and other hearing care professionals when they recommend PSAPs to patients with mild-to-moderate hearing loss.

This study has several limitations. First, the clinical experiments were only conducted on six patients diagnosed with moderate sensorineural hearing loss. Because the number of patients was so small, there was a limitation in the statistical analysis. Patients with moderate hearing loss are expected to be targeted as users of PSAPs. Nevertheless, patients with ski-slope type hearing loss or moderate-to-severe hearing loss would benefit less from the use of PSAPs, because several reports mentioned that PSAPs do not provide sufficient gain for high frequencies, so these patients have very low satisfaction with PSAPs [18]. Therefore, further investigations will need to include more participants with various types of hearing loss to obtain generalizable results. Secondly, the RPSAP was adjusted using best-practice protocols. In the majority of cases, prospective users may have to adjust the PSAPs on their own. In order to estimate the benefits of PSAPs in real-world conditions, it is necessary to measure clinical outcomes under self-adjusted conditions. In actuality, an acclimatization period is necessary to identify the exact benefits of HA devices. However, this was not done in the current study. Finally, this paper presented a comparative study between the RPSAP and HA. In the near future, additional research will need to be conducted with randomized controlled trials that contribute a higher level of evidence. PSAPs fitted by audiologists provide sufficient aided audibility and similar speech recognition performance in quiet and noisy conditions compared to HA in patients with flat-type moderate hearing loss. PSAPs could serve as a feasible, budget-friendly option for those who cannot afford HAs or are seeking a lowcost introduction to amplification. 


\section{CONFLICT OF INTEREST}

No potential conflict of interest relevant to this article was reported.

\section{ACKNOWELEDGMENTS}

This research was supported by a grant of Patient-Centered Clinical Research Coordinating Center funded by the Ministry of Health \& Welfare, Republic of Korea (grant no. HI19C0481, HC19C0128).

\section{ORCID}

$\begin{array}{ll}\text { Ga-Young Kim } & \text { https://orcid.org/0000-0002-8945-4927 } \\ \text { Jong Sei Kim } & \text { https://orcid.org/0000-0002-1798-817X } \\ \text { Mini Jo } & \text { https://orcid.org/0000-0002-8197-811X } \\ \text { Hye Yoon Seol } & \text { https://orcid.org/0000-0002-7040-1884 } \\ \text { Young Sang Cho } & \text { https://orcid.org/0000-0002-4040-7206 } \\ \text { Il Joon Moon } & \text { https://orcid.org/0000-0002-3613-0734 }\end{array}$

\section{AUTHOR CONTRIBUTIONS}

Conceptualization: all authors. Data curation: GYK, JSK, MJ. Formal analysis: GYK, KSK. Funding acquisition: YSC, IJM. Methodology: all authors. Project administration: GYK, JSK, IJM. Visualization: GYK, JSK. Writing - original draft: GYK, JSK, MJ, IJM.Writing - review \& editing: GYK, JSK, HYS, IJM.

\section{REFERENCES}

1. World Health Organization. Deafness and hearing loss. Geneva:World Health Organization; 2020 [cited 2020 Apr 3].Available from: https:// www.who.int/news-room/fact-sheets/detail/deafness-and-hearingloss.

2. Loughrey DG, Kelly ME, Kelley GA, Brennan S, Lawlor BA. Association of age-related hearing loss with cognitive function, cognitive impairment, and dementia: a systematic review and meta-analysis. JAMA Otolaryngol Head Neck Surg. 2018 Feb;144(2):115-26.
3. Moon IJ, Baek SY, Cho YS. Hearing aid use and associated factors in South Korea. Medicine (Baltimore). 2015 Oct;94(42):e1580.

4. National Evidence-based Healthcare Collaborating Agency (NECA). Analysis of hearing aid benefit and barriers in hearing aid acquisition in Korea (NECA 2010-008). Seoul: NECA; 2010 [cited 2020 Apr 3]. Available from: http://gzone.kr/gzone/gZoneSearchDetailList. do? contentsId=PLC20170213095.

5. President's Council of Advisors on Science and Technology. Aging America \& hearing loss: imperative of improved hearing technologies. Washington (DC): President's Council of Advisors on Science and Technology; 2015 [cited 2020 Apr 3]. Available from: https:// obamawhitehouse.archives.gov/sites/default/files/microsites/ostp/ PCAST/PCAST\%20hearing\%20letter\%20report.pdf.

6. Lin FR. Time for a top-down approach to hearing aid affordability and accessibility. Am J Public Health. 2018 Feb;108(2):166-8.

7. Brody L, Wu YH, Stangl E. A comparison of personal sound amplification products and hearing aids in ecologically relevant test environments. Am J Audiol. 2018 Dec;27(4):581-93.

8. Cho YS, Park SY, Seol HY, Lim JH, Cho YS, Hong SH, et al. Clinical performance evaluation of a personal sound amplification product vs a basic hearing aid and a premium hearing aid. JAMA Otolaryngol Head Neck Surg. 2019 Jun;145(6):516-22.

9. Choi JE, Kim J, Yoon SH, Hong SH, Moon IJ. A personal sound amplification product compared to a basic hearing aid for speech intelligibility in adults with mild-to-moderate sensorineural hearing loss. J Audiol Otol. 2020 Apr;24(2):91-8.

10. Callaway SL, Punch JL.An electroacoustic analysis of over-the-counter hearing aids. Am J Audiol. 2008 Jun;17(1):14-24.

11. Reed NS, Betz J, Lin FR, Mamo SK. Pilot electroacoustic analyses of a sample of direct-to-consumer amplification products. Otol Neurotol. 2017 Jul;38(6):804-8.

12. Lee JH. Pure-tone audiometry: methods for air and bone conduction. In: the Korean Audiological Society, editor. Practical manual of hearing tests. 2nd ed. Seoul: Hakjisa; 2017. p.110.

13. American Speech-Language-Hearing Association. Guidelines for manual pure-tone threshold audiometry. Rockville (MD): American Speech-Language-Hearing Association; 1977.

14. Kim JS, Lim DH, Hong HN, Shin HW, Lee KD, Hong BN, et al. Development of Korean Standard Monosyllabic Word Lists for Adults (KS-MWL-A). Audiology. 2008 Dec;4(2):126-40.

15. Moon SK, Hee Kim S, Ah Mun H, Jung HK, Lee JH, Choung YH, et al. The Korean hearing in noise test. Int J Audiol. 2008 Jun;47(6): 375-6.

16. Chan ZY, McPherson B. Over-the-counter hearing aids: a lost decade for change. Biomed Res Int. 2015;2015:827463.

17. Mamo SK, Reed NS, Nieman CL, Oh ES, Lin FR. Personal sound amplifiers for adults with hearing loss. Am J Med. 2016 Mar;129(3): 245-50.

18. Cheng CM, McPherson B. Over-the-counter hearing aids: electroacoustic characteristics and possible target client groups. Audiology. 2000 Mar-Apr;39(2):110-6. 